\title{
Calculus of variations on time scales with nabla derivatives of exponential function
}

\author{
Jie Bai ${ }^{1}\left(\mathbb{1}\right.$, Ling Bai $^{2}\left(\mathbb{D}\right.$, Zhijun Zeng $^{* 3}$ (1) \\ ${ }^{1}$ School of Mathematics, Liaoning University, Shenyang 110036, China \\ ${ }^{2}$ Website Integrated Management Department, Beijing Municipal Commission Of Health And Family \\ Planning Information Center, Beijing 100034, China \\ ${ }^{3}$ School of Mathematics and Statistics, Northeast Normal University, Changchun 130024, China
}

\begin{abstract}
In this paper, we study the calculus of variations of the nabla notion on time scales including $\nabla$-derivative, $\nabla$-integral, and $\nabla$-derivatives of exponential function. The EulerLagrange equations of the first-order both single-variable problem and multivariable problem with nabla derivatives of exponential function on time scales are obtained. In particular, we show that the calculus of variations with multiple variables could solve the problem of conditional extreme value. Moreover, we verify the solution to the multivariable problem is exactly the extremum pair. As applications of these results, an example of conditional extremum is provided.
\end{abstract}

Mathematics Subject Classification (2010). 35Q31

Keywords. time scales, the Euler-Lagrange equation, $\nabla$-derivatives of exponential function, calculus of variations, conditional extremum

\section{Introduction}

Regarded as a model of time, the time scale has turned into a newly independent theory combining difference and differential equations [11], which has been applied into several fields, such as, the calculus of variations [2-4,7,10,14,15,17,19-21], control fields $[5,6,8,9,12,18,22-24]$, and other fields involving economics [16], finance, engineering, biology and physics $[1,11]$.

In [4], the authors obtained an Euler-Lagrange equation for a first-order single-variable problem involving nabla derivatives, and set up a simple consumption and saving model of the household. More precisely, they gave a following theorem:

Theorem 1.1. (see [4], Theorem 3.1) If a function $y(t)$ provides a local extremum to the functional

$$
J[y]=\int_{\rho^{2}(a)}^{\rho(b)} L\left(t, y(\rho(t)), y^{\nabla}(t)\right) \nabla t
$$

\footnotetext{
*Corresponding Author.

Email addresses: linlanfaith@aliyun.com (J. Bai), bailing@bjchfp.gov.cn (L. Bai), zthzzj@amss.ac.cn (Z.J. Zeng)

Received: 07.03.2017; Accepted: 11.09.2018
} 
where $y \in C^{2}\left[\rho^{2}(a), \rho(b)\right]$ and $y\left(\rho^{2}(a)\right)=A, y(\rho(b))=B$, then $y$ must satisfy the EulerLagrange equation

$$
L_{y^{\rho}}\left(t, y^{\rho}, y^{\nabla}\right)-L_{y^{\nabla}}^{\nabla}\left(t, y^{\rho}, y^{\nabla}\right)=0
$$

for $t \in[\rho(a), b]$.

Furthermore, the authors stated the theorem for the functional with several variables, and set up dynamic optimization problem in economics to maximize its lifetime utility:

subject to constraint

$$
U=\int_{0}^{\sigma(T)} u(C(\rho(s))) \hat{e}_{-\delta}(\rho(s), 0) \nabla s
$$

$$
A^{\nabla}(s)=r A(\rho(s))+Y(\rho(s))-C(\rho(s)), s \in[\sigma(0), T] .
$$

In order to enrich and improve the calculus of variations on time scales, a nabla exponential function is introduced into (1.1) and a fundamental problem of the calculus of variations on time scales is actually concentrated on the Euler-Lagrange equation of the functional:

$$
F[y]=\int_{\rho^{2}(a)}^{\rho(b)} L\left(t, y(\rho(t)),\left(e_{\alpha}(t, 0) y(t)\right)^{\nabla}\right) \nabla t .
$$

This is in fact our main motivation for the present study. To be more precise, we will pay particular attention to the Euler-Lagrange equations for the calculus of variations on time scales involving the nabla notion (i.e. $\nabla$-derivative, $\nabla$-integral, $\nabla$-derivatives of exponential function).

Throughout this paper, a knowledge of time scales and time scale notation is assumed. For an excellent introduction to the calculus on time scales, we refer the readers to monographs $[10,11]$. In what follows, $\mathbb{R}$ denotes the set of real numbers, and $\mathbb{Z}$ denotes the set of integers. A time scale $\mathbb{T}$ is an arbitrary nonempty closed subset of $\mathbb{R}$. Note that $[a, b]_{\mathcal{K}}=[a, b]$ if $a$ is right-dense and $[a, b]_{\mathcal{K}}=[\sigma(a), b]$ if $a$ is right-scattered. The admissible functions $y$ are of class

$$
C^{s}([a, b], \mathbb{R}) \triangleq\left\{y:[a, b] \cap \mathbb{T} \rightarrow \mathbb{R} \mid y^{\nabla^{s}} \text { is continuous on }[a, b]_{\mathcal{K}^{s}}\right\},
$$

where $[a, b] \cap \mathbb{T}$ has at least $s+1$ points.

Our main results of the paper are as follows.

Theorem 1.2. For the problem

$$
F[y]=\int_{\rho^{2}(a)}^{\rho(b)} L\left(t, y(\rho(t)),\left(e_{\alpha}(t, 0) y(t)\right)^{\nabla}\right) \nabla t \rightarrow \operatorname{extr}, \quad y\left(\rho^{2}(a)\right)=A, y(\rho(b))=B .
$$

If $y(t) \in C^{1}\left(\left[\rho^{2}(a), b\right], \mathbb{R}\right)$ is a weak local extremum point, then the following Euler-Lagrange equation

$L_{y^{\rho}}\left(t, y^{\rho},\left(e_{\alpha}(t, 0) y(t)\right)^{\nabla}\right)-L_{\left(e_{\alpha}(t, 0) y(t)\right) \nabla}^{\nabla}\left(t, y^{\rho},\left(e_{\alpha}(t, 0) y(t)\right)^{\nabla}\right) e_{\alpha}(\rho(t), 0)=0, t \in\left[\rho^{2}(a), b\right]_{\mathcal{K}}$, holds.

For the first-order multivariable problem, the corresponding result stated as follows.

Theorem 1.3. For the multivariable problem

$$
\begin{aligned}
F\left[y_{1}, \ldots, y_{n}, u_{1}, \ldots, u_{m}\right]= & \int_{\rho^{2}(a)}^{\rho(b)} L\left(t, y_{1}^{\rho}, \ldots, y_{n}^{\rho},\left(e_{\alpha}(t, 0) y_{1}(t)\right)^{\nabla}, \ldots,\left(e_{\alpha}(t, 0) y_{n}(t)\right)^{\nabla},\right. \\
& \left.u_{1}^{\rho}, \ldots, u_{m}^{\rho},\left(u_{1}(t)\right)^{\nabla}, \ldots,\left(u_{m}(t)\right)^{\nabla}\right) \nabla t \rightarrow \operatorname{extr}, \\
& y_{i}\left(\rho^{2}(a)\right)=u_{j}\left(\rho^{2}(a)\right)=A, \quad y_{i}(\rho(b))=u_{j}(\rho(b))=B, \\
& i=1,2, \ldots, n, \quad j=1, \ldots, m .
\end{aligned}
$$


If $y_{i}(t), u_{j}(t) \in C^{1}\left(\left[\rho^{2}(a), b\right], \mathbb{R}\right)$ are weak local extremum points, then the Euler-Lagrange equations

$$
\begin{aligned}
& L_{y_{i}^{\rho}}-L_{\left(e_{\alpha}(t, 0) y_{i}\right) \nabla}^{\nabla} \cdot e_{\alpha}(\rho(t), 0)=0, \\
& L_{u_{j}^{\rho}}-L_{u_{j}^{\nabla}}^{\nabla}=0
\end{aligned}
$$

hold.

Remark 1.4. Theorem 1.3 can also be applied to solve conditional extremum problems. We refer the reader to the details of Corollary 2.2 in Section 2. In this paper, optimal consumption path (see the example in Section 3 ) is obtained through a conditional extremum for which Theorem 1.3 is applicable.

Theorem 1.5. The solution to the multivariable problem (1.2) is the optimal solution, for linearity of $f$ and concavity of $L$ in $\left(y^{\rho}(t), u^{\rho}(t)\right), t \in\left[\rho^{2}(a), b\right]_{\mathcal{K}}$. If $(y(t), u(t)) \in$ $C^{1}\left(\left[\rho^{2}(a), b\right], \mathbb{R}\right) \times C^{1}\left(\left[\rho^{2}(a), b\right], \mathbb{R}\right)$ is the solution pair of the multi-variable problem $(1.2)$, for linearity of $f$ and concavity of $L$ in $\left(y^{\rho}(t), u^{\rho}(t)\right), t \in\left[\rho^{2}(a), b\right]_{\mathcal{K}}$, then $(y(t), u(t))$ must be precisely the extremum pair and satisfy the Euler-Lagrange system (1.3).

As an example, we consider an economical model on time scale. The purpose is to find a consumption path that would maximize utility $U$ of a family:

$$
U=\int_{0}^{T} u(C(\rho(s))) e_{-\beta}(\rho(s), 0) \cdot \frac{L(\rho(s))}{H} \nabla s,
$$

subject to constraint

$$
\begin{aligned}
{\left[k(s) e_{n} \bigoplus g(s, 0)\right]^{\nabla}=} & \frac{1}{\nu(s)} \cdot k(\rho(s)) \cdot e_{n} \bigoplus g(\rho(s), 0) \cdot\left(e_{r}(s, \rho(s))-1\right) \\
& +\frac{1}{\nu(s)} \cdot e_{n} \bigoplus g(\rho(s), 0) \cdot e_{r}(s, \rho(s)) \cdot \omega(\rho(s)) \\
& -\frac{1}{\nu(s)} \cdot \frac{1}{A_{0}} e_{n}(\rho(s), 0) \cdot e_{r}(s, \rho(s)) \cdot C(\rho(s)),
\end{aligned}
$$

for $s \in[0, \sigma(T)]$.

By Theorem 1.3 and the chain rule, we have

$$
\left\{\frac{2-[s-\rho(t)]}{[1-\nu(t) \cdot(-\beta \oplus r)] \cdot[s-\rho(t)]}-\frac{1}{(1+\nu(t) \beta) \cdot(1-\nu(t) r) \cdot e_{r}(t, s) \cdot[s-\rho(t)]}\right\} \cdot \frac{1}{C^{\nabla}(t)}<0 .
$$

In the above example, the economic implications of expression and symbol are explained in Section 3. Consumption and saving decisions can be established by discussing (1.4). We refer the reader to Section 3 for details.

This paper is organized as follows. In Section 2, we study calculus of variations for the time scale case by using the nabla notion (i.e. $\nabla$-derivative, $\nabla$-integral, $\nabla$-derivatives of exponential function). The Euler-Lagrange equation of the calculus of variations of the first-order single-variable problem on time scales will be proved. Furthermore, we obtain the Euler-Lagrange system of the first-order multivariable problem, and show that calculus of variations with multiple variables could be applied into conditional extremum problems. In addition, we verify that the solution to the multivariable problem is quite right the extremal solution. Theorems 1.2-1.5 will be proved in this section. In Section 3, we demonstrate applications of our main results by considering an example of maximizing utility in which an optimal consumption path is observed.

\section{The proofs of main results}

Here, we first recall from [4] an equality which is derived based on the integral property. 
Lemma 2.1. (see [4], Lemma2.1) If $f(t)$ is continuous on $[\rho(a), b]$, where $\rho(a)<b$, and if

$$
\int_{\rho(a)}^{b} f(t) g(t) \nabla t=0
$$

for every function $g(t) \in C([\rho(a), b], \mathbb{R})$ with $g(\rho(a))=g(b)=0$, then $f(t)=0$ for $t \in[\rho(a), b]$.

Now, we give the proofs of our main results.

The proof of Theorem 1.2. Let $h:\left[\rho^{2}(a), b\right] \rightarrow \mathbb{R}$ be any admissible variation, i.e., $h \in C^{1}\left(\left[\rho^{2}(a), b\right], \mathbb{R}\right)$ with $h\left(\rho^{2}(a)\right)=h(\rho(b))=0$. Assume that this variational problem has a local extremum at $y$.

Define

$$
\Phi(\varepsilon)=F[y(t)+\varepsilon h(t)]
$$

where $\varepsilon \in(-\infty,+\infty)$.

Due to $\Phi$ has a local extremum at $\varepsilon=0$, it follows that

$$
\Phi^{\prime}(0)=0, \Phi^{\prime \prime}(0) \geq 0(\leq 0)
$$

in the local minimum (maximum) case.

Next we consider

$$
\begin{aligned}
\Phi(\varepsilon) & =F[y(t)+\varepsilon h(t)] \\
& =\int_{\rho^{2}(a)}^{\rho(b)} L\left(t, y(\rho(t))+\varepsilon h(\rho(t)),\left(e_{\alpha}(t, 0) y(t)\right)^{\nabla}+\varepsilon\left(e_{\alpha}(t, 0) h(t)\right)^{\nabla}\right) \nabla t .
\end{aligned}
$$

For simplicity, we denote $L\left(t, y(\rho(t))+\varepsilon h(\rho(t)),\left(e_{\alpha}(t, 0) y(t)\right)^{\nabla}+\varepsilon\left(e_{\alpha}(t, 0) h(t)\right)^{\nabla}\right)$ by $L(t, u, v)$.

Differentiating with respect to $\varepsilon$, we acquire

$$
\begin{aligned}
\Phi^{\prime}(\varepsilon)= & \int_{\rho^{2}(a)}^{\rho(b)} \frac{\mathrm{d}}{\mathrm{d} \varepsilon} L\left(t, y(\rho(t))+\varepsilon h(\rho(t)),\left(e_{\alpha}(t, 0) y(t)\right)^{\nabla}+\varepsilon\left(e_{\alpha}(t, 0) h(t)\right)^{\nabla}\right) \nabla t \\
= & \int_{\rho^{2}(a)}^{\rho(b)}\left\{L_{u}\left(t, y(\rho(t))+\varepsilon h(\rho(t)),\left(e_{\alpha}(t, 0) y(t)\right)^{\nabla}+\varepsilon\left(e_{\alpha}(t, 0) h(t)\right)^{\nabla}\right) h(\rho(t))\right. \\
& \left.+L_{v}\left(t, y(\rho(t))+\varepsilon h(\rho(t)),\left(e_{\alpha}(t, 0) y(t)\right)^{\nabla}+\varepsilon\left(e_{\alpha}(t, 0) h(t)\right)^{\nabla}\right)\left(e_{\alpha}(t, 0) h(t)\right)^{\nabla}\right\} \nabla t .
\end{aligned}
$$

It is obvious that

$$
\begin{aligned}
\Phi^{\prime}(0)= & \int_{\rho^{2}(a)}^{\rho(b)}\left\{L_{u}\left(t, y(\rho(t)),\left(e_{\alpha}(t, 0) y(t)\right)^{\nabla}\right) h(\rho(t))\right. \\
& \left.+L_{v}\left(t, y(\rho(t)),\left(e_{\alpha}(t, 0) y(t)\right)^{\nabla}\right)\left(e_{\alpha}(t, 0) h(t)\right)^{\nabla}\right\} \nabla t,
\end{aligned}
$$

and we denote the first variation of $F(y)$ by $F_{1}(h)$.

So a necessary condition for $y(t)$ to be a local minimum is

$$
\begin{aligned}
F_{1}(h)= & \int_{\rho^{2}(a)}^{\rho(b)}\left\{L_{u}\left(t, y(\rho(t)),\left(e_{\alpha}(t, 0) y(t)\right)^{\nabla}\right) h(\rho(t))\right. \\
& \left.+L_{v}\left(t, y(\rho(t)),\left(e_{\alpha}(t, 0) y(t)\right)^{\nabla}\right)\left(e_{\alpha}(t, 0) h(t)\right)^{\nabla}\right\} \nabla t \\
= & 0
\end{aligned}
$$

for all $h \in C^{1}\left(\left[\rho^{2}(a), b\right], \mathbb{R}\right)$ with $h\left(\rho^{2}(a)\right)=h(\rho(b))=0$. 
According to properties of $\nabla$-integral, it follows that

$$
\begin{aligned}
F_{1}(h)= & \int_{\rho^{2}(a)}^{\rho(a)}\left\{L_{u}\left(t, y(\rho(t)),\left(e_{\alpha}(t, 0) y(t)\right)^{\nabla}\right) h(\rho(t))\right. \\
& \left.+L_{v}\left(t, y(\rho(t)),\left(e_{\alpha}(t, 0) y(t)\right)^{\nabla}\right)\left(e_{\alpha}(t, 0) h(t)\right)^{\nabla}\right\} \nabla t \\
& +\int_{\rho(a)}^{\rho(b)}\left\{L_{u}\left(t, y(\rho(t)),\left(e_{\alpha}(t, 0) y(t)\right)^{\nabla}\right) h(\rho(t))\right. \\
& \left.+L_{v}\left(t, y(\rho(t)),\left(e_{\alpha}(t, 0) y(t)\right)^{\nabla}\right)\left(e_{\alpha}(t, 0) h(t)\right)^{\nabla}\right\} \nabla t \\
= & \left(\rho(a)-\rho^{2}(a)\right)\left\{L_{u}\left(\rho(a), y\left(\rho^{2}(a)\right),\left(e_{\alpha}(\rho(a), 0) y(\rho(a))\right)^{\nabla}\right) h\left(\rho^{2}(a)\right)\right. \\
& \left.+L_{v}\left(\rho(a), y\left(\rho^{2}(a)\right),\left(e_{\alpha}(\rho(a), 0) y(\rho(a))\right)^{\nabla}\right)\left(e_{\alpha}(\rho(a), 0) h(\rho(a))\right)^{\nabla}\right\} \\
& +\int_{\rho(a)}^{\rho(b)}\left\{L_{u}\left(t, y(\rho(t)),\left(e_{\alpha}(t, 0) y(t)\right)^{\nabla}\right) h(\rho(t))\right. \\
& \left.+L_{v}\left(t, y(\rho(t)),\left(e_{\alpha}(t, 0) y(t)\right)^{\nabla}\right)\left(e_{\alpha}(t, 0) h(t)\right)^{\nabla}\right\} \nabla t \\
= & \left(\rho(a)-\rho^{2}(a)\right) L_{v}\left(\rho(a), y\left(\rho^{2}(a)\right),\left(e_{\alpha}(\rho(a), 0) y(\rho(a))\right)^{\nabla}\right)\left(e_{\alpha}(\rho(a), 0) h(\rho(a))\right)^{\nabla} \\
& +\int_{\rho(a)}^{\rho(b)}\left\{L_{u}\left(t, y(\rho(t)),\left(e_{\alpha}(t, 0) y(t)\right)^{\nabla}\right) h(\rho(t))\right. \\
& \left.+L_{v}\left(t, y(\rho(t)),\left(e_{\alpha}(t, 0) y(t)\right)^{\nabla}\right)\left(e_{\alpha}(t, 0) h(t)\right)^{\nabla}\right\} \nabla t .
\end{aligned}
$$

Thanks to the equality

$$
\left(\rho(a)-\rho^{2}(a)\right)\left(e_{\alpha}(\rho(a), 0) h(\rho(a))\right)^{\nabla}=e_{\alpha}(\rho(a), 0) h(\rho(a))-e_{\alpha}\left(\rho^{2}(a), 0\right) h\left(\rho^{2}(a)\right),
$$

we obtain

$$
\begin{aligned}
F_{1}(h)= & L_{v}\left(\rho(a), y\left(\rho^{2}(a)\right),\left(e_{\alpha}(\rho(a), 0) y(\rho(a))\right)^{\nabla}\right) e_{\alpha}(\rho(a), 0) h(\rho(a)) \\
& +\int_{\rho(a)}^{\rho(b)}\left\{L_{u}\left(t, y(\rho(t)),\left(e_{\alpha}(t, 0) y(t)\right)^{\nabla}\right) h(\rho(t))\right. \\
& \left.+L_{v}\left(t, y(\rho(t)),\left(e_{\alpha}(t, 0) y(t)\right)^{\nabla}\right)\left(e_{\alpha}(t, 0) h(t)\right)^{\nabla}\right\} \nabla t .
\end{aligned}
$$

By virtue of the formula (see [11], Theorem 8.47), it follows that

$$
\begin{aligned}
F_{1}(h)= & L_{v}\left(\rho(a), y\left(\rho^{2}(a)\right),\left(e_{\alpha}(\rho(a), 0) y(\rho(a))\right)^{\nabla}\right) e_{\alpha}(\rho(a), 0) h(\rho(a)) \\
& +\int_{\rho(a)}^{\rho(b)} L_{u}\left(t, y(\rho(t)),\left(e_{\alpha}(t, 0) y(t)\right)^{\nabla}\right) h(\rho(t)) \nabla t \\
& +L_{v}\left(\rho(b), y\left(\rho^{2}(b)\right),\left(e_{\alpha}(\rho(b), 0) y(\rho(b))\right)^{\nabla}\right)\left(e_{\alpha}(\rho(b), 0) h(\rho(b))\right) \\
& -L_{v}\left(\rho(a), y\left(\rho^{2}(a)\right),\left(e_{\alpha}(\rho(a), 0) y(\rho(a))\right)^{\nabla}\right) e_{\alpha}(\rho(a), 0) h(\rho(a)) \\
& -\int_{\rho(a)}^{\rho(b)} L_{v}^{\nabla}\left(t, y(\rho(t)),\left(e_{\alpha}(t, 0) y(t)\right)^{\nabla}\right)\left(e_{\alpha}(\rho(t), 0) h(\rho(t))\right) \nabla t \\
= & \int_{\rho(a)}^{\rho(b)}\left\{L_{u}\left(t, y(\rho(t)),\left(e_{\alpha}(t, 0) y(t)\right)^{\nabla}\right) h(\rho(t))\right. \\
& \left.-L_{v}^{\nabla}\left(t, y(\rho(t)),\left(e_{\alpha}(t, 0) y(t)\right)^{\nabla}\right) e_{\alpha}(\rho(t), 0) h(\rho(t))\right\} \nabla t .
\end{aligned}
$$

Again, using the property of $\nabla$-integral, we have

$$
\begin{aligned}
& \int_{\rho(b)}^{b}\left\{L_{u}\left(t, y(\rho(t)),\left(e_{\alpha}(t, 0) y(t)\right)^{\nabla}\right)-L_{v}^{\nabla}\left(t, y(\rho(t)),\left(e_{\alpha}(t, 0) y(t)\right)^{\nabla}\right) e_{\alpha}(\rho(t), 0)\right\} h(\rho(t)) \nabla t \\
& =(b-\rho(b))\left\{L_{u}\left(b, y(\rho(b)),\left(e_{\alpha}(b, 0) y(b)\right)^{\nabla}\right)-L_{v}^{\nabla}\left(b, y(\rho(b)),\left(e_{\alpha}(b, 0) y(b)\right)^{\nabla}\right) e_{\alpha}(\rho(b), 0)\right\} h(\rho(b)) \\
& =0
\end{aligned}
$$


and

$\int_{\rho(a)}^{b}\left\{L_{u}\left(t, y(\rho(t)),\left(e_{\alpha}(t, 0) y(t)\right)^{\nabla}\right)-L_{v}^{\nabla}\left(t, y(\rho(t)),\left(e_{\alpha}(t, 0) y(t)\right)^{\nabla}\right) e_{\alpha}(\rho(t), 0)\right\} h(\rho(t)) \nabla t=0$, for all $h \in C^{1}\left(\left[\rho^{2}(a), b\right], \mathbb{R}\right)$ with $h\left(\rho^{2}(a)\right)=h(\rho(b))=0$. According to Lemma 2.1, we conclude that

$$
L_{y^{\rho}}\left(t, y^{\rho},\left(e_{\alpha}(t, 0) y(t)\right)^{\nabla}\right)-L_{\left(e_{\alpha}(t, 0) y(t)\right)^{\nabla}}^{\nabla}\left(t, y^{\rho},\left(e_{\alpha}(t, 0) y(t)\right)^{\nabla}\right) e_{\alpha}(\rho(t), 0)=0,
$$

for $t \in[\rho(a), b]$, exactly speaking for $t \in\left[\rho^{2}(a), b\right]_{\mathcal{K}}$. This completes the proof.

The proof of Theorem 1.3 follows from the same argument as that of Theorem 1.2 and we omit it.

Corollary 2.2. If a pair $(y(t), u(t))$ provide an extremum to the functional

$$
F[y, u]=\int_{\rho^{2}(a)}^{\rho(b)} L\left(t, y^{\rho}(t), u^{\rho}(t)\right) \nabla t
$$

among all pairs $(y, u)$ such that

$$
\left(e_{\alpha}(t, 0) y(t)\right)^{\nabla}=f\left(t, y^{\rho}(t), u^{\rho}(t)\right),
$$

then, $(y(t), u(t))$ must satisfy the Euler-Lagrange system

$$
\begin{aligned}
& L_{y^{\rho}}\left(t, y^{\rho}(t), u^{\rho}(t)\right)+\lambda^{\rho}\left[f_{y^{\rho}}\left(t, y^{\rho}(t), u^{\rho}(t)\right)=-\left(\lambda^{\rho}\right)^{\nabla} \cdot e_{\alpha}(\rho(t), 0),\right. \\
& L_{u^{\rho}}\left(t, y^{\rho}(t), u^{\rho}(t)\right)+\lambda^{\rho}\left[f_{u^{\rho}}\left(t, y^{\rho}(t), u^{\rho}(t)\right)=0,\right. \\
& f\left(t, y^{\rho}(t), u^{\rho}(t)\right)-\left(e_{\alpha}(t, 0) y(t)\right)^{\nabla}=0 .
\end{aligned}
$$

Proof. Firstly, we introduce a multiplier $\lambda(t)$, such that $\lambda^{\rho}(t) \in C^{1}\left(\left[\rho^{2}(a), b\right], \mathbb{R}\right)$. Secondly, we consider the functional

$F^{*}\left[y, u, \lambda,\left(e_{\alpha}(t, 0) y(t)\right)^{\nabla}\right]=\int_{\rho^{2}(a)}^{\rho(b)}\left[L\left(t, y^{\rho}(t), u^{\rho}(t)\right)+\lambda^{\rho}(t)\left(f\left(t, y^{\rho}(t), u^{\rho}(t)\right)-\left(e_{\alpha}(t, 0) y(t)\right)^{\nabla}\right)\right] \nabla t$.

Finally, we set

$G\left(t, u^{\rho}, y^{\rho}, \lambda^{\rho}, u^{\nabla},\left(e_{\alpha}(t, 0) y(t)\right)^{\nabla}\right)=L\left(t, y^{\rho}(t), u^{\rho}(t)\right)+\lambda^{\rho}(t)\left[f\left(t, y^{\rho}(t), u^{\rho}(t)\right)-\left(e_{\alpha}(t, 0) y(t)\right)^{\nabla}\right]$.

By Theorem 1.3, it is obvious that the Euler-Lagrange system can be written as

$$
\begin{aligned}
G_{y^{\rho}} & =G_{\left(e_{\alpha}(t, 0) y(t)\right)^{\nabla}}^{\nabla} \cdot e_{\alpha}(\rho(t), 0), \\
G_{u^{\rho}} & =G_{u^{\nabla}}^{\nabla}, \\
G_{\lambda^{\rho}} & =G_{\lambda^{\nabla}}^{\nabla} .
\end{aligned}
$$

More concretely,

$$
\begin{aligned}
& L_{y^{\rho}}\left(t, y^{\rho}(t), u^{\rho}(t)\right)+\lambda^{\rho}\left[f_{y^{\rho}}\left(t, y^{\rho}(t), u^{\rho}(t)\right)=-\left(\lambda^{\rho}\right)^{\nabla} \cdot e_{\alpha}(\rho(t), 0),\right. \\
& L_{u^{\rho}}\left(t, y^{\rho}(t), u^{\rho}(t)\right)+\lambda^{\rho}\left[f_{u^{\rho}}\left(t, y^{\rho}(t), u^{\rho}(t)\right)=0,\right. \\
& f\left(t, y^{\rho}(t), u^{\rho}(t)\right)-\left(e_{\alpha}(t, 0) y(t)\right)^{\nabla}=0,
\end{aligned}
$$

hold.

The proof of Theorem 1.5. Assume that the pair $(y, u)$ satisfies all the optimality conditions, and let $(\bar{y}, \bar{u})$ be another admissible pair. Owing to the hypotheses of linearity 
and concavity, we measure the difference

$$
\begin{aligned}
|F[\bar{y}, \bar{u}]-F[y, u]|= & \int_{\rho^{2}(a)}^{\rho(b)}\left|L\left(t, \bar{y}^{\rho}, \bar{u}^{\rho}\right)-L\left(t, y^{\rho}, u^{\rho}\right)\right| \nabla t \\
\leq & \int_{\rho^{2}(a)}^{\rho(b)}\left\{L_{u}\left(t, y^{\rho}, u^{\rho}\right)\left(\bar{y}^{\rho}-y^{\rho}\right)+L_{v}\left(t, y^{\rho}, u^{\rho}\right)\left(\bar{u}^{\rho}-u^{\rho}\right)\right\} \nabla t \\
= & \int_{\rho^{2}(a)}^{\rho(b)} \mid \lambda^{\rho}\left[f_{y^{\rho}}\left(t, y^{\rho}, u^{\rho}\right)\left(\bar{y}^{\rho}-y^{\rho}\right)+f_{u^{\rho}}\left(t, y^{\rho}, u^{\rho}\right)\left(\bar{u}^{\rho}-u^{\rho}\right)\right] \\
& +\left(\lambda^{\rho}\right) e_{\alpha}(\rho(t), 0)\left(\bar{y}^{\rho}-y^{\rho}\right) \mid \nabla t \\
= & \int_{\rho^{2}(a)}^{\rho(b)} \mid \lambda^{\rho}\left[f_{y^{\rho}}\left(t, y^{\rho}, u^{\rho}\right)\left(\bar{y}^{\rho}-y^{\rho}\right)+f_{u^{\rho}}\left(t, y^{\rho}, u^{\rho}\right)\left(\bar{u}^{\rho}-u^{\rho}\right)\right] \\
& +\left[\left(\lambda^{\rho} e_{\alpha}(t, 0) \bar{y}\right)\right]^{\nabla}-\lambda^{\rho}\left(e_{\alpha}(t, 0) \bar{y}\right)^{\nabla}-\left[\left(\lambda^{\rho} e_{\alpha}(t, 0) y\right)\right]^{\nabla}+\lambda^{\rho}\left(e_{\alpha}(t, 0) y\right)^{\nabla} \mid \nabla t \\
= & \int_{\rho^{2}(a)}^{\rho(b)} \mid \lambda^{\rho}\left[f_{y^{\rho}}\left(t, y^{\rho}, u^{\rho}\right)\left(\bar{y}^{\rho}-y^{\rho}\right)+f_{u^{\rho}}\left(t, y^{\rho}, u^{\rho}\right)\left(\bar{u}^{\rho}-u^{\rho}\right)\right] \\
& +\lambda^{\rho}\left(e_{\alpha}(t, 0) y\right)^{\nabla}-\lambda^{\rho}\left(e_{\alpha}(t, 0) \bar{y}\right)^{\nabla}+\left[\lambda^{\rho} e_{\alpha}(t, 0) \bar{y}\right]^{\nabla}-\left[\lambda^{\rho} e_{\alpha}(t, 0) y\right] \nabla \mid \nabla t \\
\leq & \int_{\rho^{2}(a)}^{\rho(b)} \mid \lambda^{\rho}\left[f_{y^{\rho}}\left(t, y^{\rho}, u^{\rho}\right)\left(\bar{y}^{\rho}-y^{\rho}\right)+f_{u^{\rho}}\left(t, y^{\rho}, u^{\rho}\right)\left(\bar{u}^{\rho}-u^{\rho}\right)\right. \\
& \left.+f\left(t, y^{\rho}, u^{\rho}\right)-f\left(t, \bar{y}^{\rho}, \bar{u}^{\rho}\right)\right]|+|\left[\lambda^{\rho} e_{\alpha}(t, 0) \bar{y}\right]^{\nabla}-\left[\lambda^{\rho} e_{\alpha}(t, 0) y\right]^{\nabla} \mid \nabla t \\
= & \int_{\rho^{2}(a)}^{\rho(b)} 0 \nabla t+\int_{\rho^{2}(a)}^{\rho(b)}\left[\left(\lambda^{\rho} e_{\alpha}(t, 0)\right)(\bar{y}-y)\right]^{\nabla} \nabla t \\
= & \left.\left(\lambda^{\rho} e_{\alpha}(t, 0)\right)(\bar{y}-y)\right|_{\rho^{2}(a)} ^{\rho(b)} \\
= & 0,
\end{aligned}
$$

for $\bar{y}, y \in C^{1}\left(\left[\rho^{2}(a), b\right], \mathbb{R}\right)$, and $y\left(\rho^{2}(a)\right)=A, y(\rho(b))=B$. This concludes that $(y, u)$ is indeed unique and completes the proof.

\section{An economic application of conditional extremum}

In this section, we will provide a conditional extremum in economics to illustrate an important role played by Theorem 1.3.

The income of a household usually comes from the labor and capital, beside of these, some from the profits of the firms. We suppose that the utility $U$ of a family is

$$
U=\int_{0}^{T} u(C(\rho(s))) e_{-\beta}(\rho(s), 0) \cdot \frac{L(\rho(s))}{H} \nabla s,
$$

where $C(\rho(s))$ is the consumption of each member of the household at time $\rho(s), u(\cdot)$ is each member's utility, $L(\rho(s))$ is the total population of the economy, $H$ is the number of households, $\frac{L(\rho(s))}{H}$ is the number of members of the household, $\beta$ is the discount rate, the greater is $\beta$, the less the household values future consumption relative to current consumption (see [13]).

Our position is to maximize $U$ under the household's capital holdings:

$\frac{K(s)}{H}=\frac{K(\rho(s))}{H} \cdot e_{r}(s, \rho(s))+\frac{W(\rho(s)) L(\rho(s))}{H} \cdot e_{r}(s, \rho(s))-\frac{C(\rho(s)) L(\rho(s))}{H} \cdot e_{r}(s, \rho(s))$, where $K(t)$ is the amount of capital at $t$ in the economy, and $K(t)=k(t) A(t) L(t), A(t)$ is the knowledge at $t$ in the economy, $r$ is the real interest rate, $e_{r}(t, s)$ shows the effects of continuously compounding interest over the period $[s, t]$, and $\omega(t)$ is the wage of each member of the household at time $\rho(s)$. Labor and knowledge functions satisfy: $L^{\nabla}(t)=$ $n L(t), A^{\nabla}(t)=g A(t)$, where $n$ and $g$ are parameters. 
After calculation on time scales, the constraint reduces to

$$
\begin{aligned}
{\left[k(s) e_{n} \bigoplus g(s, 0)\right]^{\nabla}=} & \frac{1}{\nu(s)} \cdot k(\rho(s)) \cdot e_{n} \bigoplus g(\rho(s), 0) \cdot\left(e_{r}(s, \rho(s))-1\right) \\
& +\frac{1}{\nu(s)} \cdot e_{n} \bigoplus g(\rho(s), 0) \cdot e_{r}(s, \rho(s)) \cdot \omega(\rho(s)) \\
& -\frac{1}{\nu(s)} \cdot \frac{1}{A_{0}} e_{n}(\rho(s), 0) \cdot e_{r}(s, \rho(s)) \cdot C(\rho(s)),
\end{aligned}
$$

$s \in[0, \sigma(T)]$, where $A_{0}$ is the initial levels of knowledge.

In this model:

$$
\begin{aligned}
G\left(s, C, k,\left[k(s) e_{n} \bigoplus g\right.\right. & \left.(s, 0)]^{\nabla}\right)=u(C(\rho(s))) e_{-\beta}(\rho(s), 0) \cdot \frac{L(\rho(s))}{H} \\
& +\lambda(\rho(s))\left\{\frac{1}{\nu(s)} \cdot k(\rho(s)) \cdot e_{n} \bigoplus g(\rho(s), 0) \cdot\left(e_{r}(s, \rho(s))-1\right)\right. \\
& +\frac{1}{\nu(s)} \cdot e_{n} \bigoplus g(\rho(s), 0) \cdot e_{r}(s, \rho(s)) \cdot \omega(\rho(s)) \\
& \left.-\frac{1}{\nu(s)} \cdot \frac{1}{A_{0}} e_{n}(\rho(s), 0) \cdot e_{r}(s, \rho(s)) \cdot C(\rho(s))-\left[k(s) e_{n} \bigoplus g(s, 0)\right]^{\nabla}\right\} .
\end{aligned}
$$

By Corollary 2.2, Euler-Lagrange equations are

$$
\begin{aligned}
& u^{\prime}(C(\rho(s))) \cdot e_{-\beta}(\rho(s), 0) \frac{L_{0} A_{0}}{H}-\lambda(\rho(s)) \cdot \frac{1}{\nu(s)} \cdot e_{r}(s, \rho(s))=0, \\
& \lambda(\rho(s)) \cdot \frac{1}{\nu(s)} \cdot\left(e_{r}(s, \rho(s))-1\right)=-[\lambda(\rho(s))]^{\nabla} .
\end{aligned}
$$

Without loss of generality, we can replace $\rho(s)$ by $t$ and obtain the following dynamic equation:

$$
\frac{\left(u^{\prime}(C(t))\right)^{\nabla}}{u^{\prime}(C(t))}=\frac{2-[s-\rho(t)]}{[1-\nu(t) \cdot(-\beta \oplus r)] \cdot[s-\rho(t)]}-\frac{1}{(1+\nu(t) \beta) \cdot(1-\nu(t) r) \cdot e_{r}(t, s) \cdot[s-\rho(t)]} .
$$

Using the chain rule (see [4], Theorem 2.6), we obtain

$$
\begin{aligned}
\frac{\left(u^{\prime}(C(t))\right)^{\nabla}}{u^{\prime}(C(t))}= & \frac{\left[\int_{0}^{1} u^{\prime \prime}\left(C(t)+h \nu(t) C^{\nabla}(t)\right) \mathrm{d} h\right] \cdot C^{\nabla}(t)}{u^{\prime}(C(t))} \\
= & \frac{2-[s-\rho(t)]}{[1-\nu(t) \cdot(-\beta \oplus r)] \cdot[s-\rho(t)]} \\
& -\frac{1}{(1+\nu(t) \beta) \cdot(1-\nu(t) r) \cdot e_{r}(t, s) \cdot[s-\rho(t)]} \\
:= & H(s, t) .
\end{aligned}
$$

We can treat $\frac{\left(u^{\prime}(C(t))\right)^{\nabla}}{u^{\prime}(C(t))}$ as the growth rate of marginal utility. Next we have

$$
H(s, t) \cdot \frac{1}{C^{\nabla}(t)}<0
$$

if the utility function is concave $\left(u^{\prime}>0, u^{\prime \prime}<0\right)$. The last inequality shows the growth rate of consumption $C^{\nabla}(t)$ falls into two aspects:

(1) If $H(s, t)<0$, then $C^{\nabla}(t)>0$, which indicates the consumer will consume less currently and more in the future. Especially, when $s-\rho(t)>2$, it is clear that $C^{\nabla}(t)>0$. That is to say the consumer is not eager to consume at present but make some saving to pass the savings on to the consumption of more than two periods in the future. 
(2) If $H(s, t)>0$, then $C^{\nabla}(t)<0$, which implies the consumer is impatient and will consume more in the earlier periods and less in the future periods. Especially, when $2-[s-\rho(t)]$ is close to 2 , it is obvious that $C^{\nabla}(t)<0$. That is to say the consumer will consume more at $\rho(t)$. In fact, the time interval is very short $(s-\rho(t)$ is close to 0$)$, and the consumer can also get wages or income in the next moment $t$ and $s$ to determine future savings and consumption.

Remark 3.1. 1 is one period. $\beta$ and $r$ are the discount rate and the interest rate respectively of one period and fixed in one period. $\nu(t)=t-\rho(t)$ may not be in one period.

Remark 3.2. Because of $e_{p}(\rho(t), s)=(1-\nu(t) p(t)) e_{p}(t, s), e_{p}(\rho(t), s)>0$, and $e_{p}(t, s)>0$ (see [11]), $1-\nu(t) p(t)>0$ can be easily obtained. Hence, it is clear that $1-\nu(t)(-\beta \oplus r)>0$, $1+\nu(t) \beta>0$, and $1-\nu(t) r>0$.

Therefore, given utility function $u(C)$, specific parameter values for $\beta, r$, a backward jump operator $\nu(t)$, and a forward jump operator $\mu(t)(\mu(t)=\sigma(t)-t)$, we can establish a dynamic system of consumption on time scales.

In special, if $\mathbb{T}=\mathbb{R}$, the model degenerates into continuous model:

$$
U=\int_{0}^{T} u(C(s)) e^{-\beta s} \frac{L(s)}{H} d s,
$$

subject to the constraint:

$$
\left[e^{(n+g) s} k(s)\right]^{\prime}=k(t) e^{(n+g) t}\left(e^{r(s-t)}-1\right)+e^{(n+g) t} \omega(t) e^{r(s-t)}-\frac{e^{n t}}{A_{0}} e^{r(s-t)} C(t) ;
$$

if $\mathbb{T}=\mathbb{Z}$, the model degenerates into discrete model:

$$
U=\sum_{s=0}^{T}\left(\frac{1}{1+\beta}\right)^{s} u(C(s)) \frac{L(s)}{H},
$$

subject to the constraint:

$$
\frac{K(s+1)}{H}=\frac{1}{H} \cdot K(s) \cdot \frac{1}{1-r}+\frac{1}{H} \cdot W(s) L(s) \cdot \frac{1}{1-r}-\frac{1}{H} \cdot C(s) L(s) \cdot \frac{1}{1-r},
$$

that is,

$$
K(s+1)=K(s) \cdot \frac{1}{1-r}+W(s) L(s) \cdot \frac{1}{1-r}-C(s) L(s) \cdot \frac{1}{1-r} .
$$

\section{Conclusions}

The first conclusion is that we obtain necessary conditions for exponential function with $\nabla$-derivative, $\nabla$-integral. In the special case $\alpha=0$, Theorem 1.2 and Theorem 1.3 cover the results [see [4], Theorem 3.1] and [see [4], Theorem 3.2], respectively. Secondly, nabla exponential function is more efficient for application of economic optimization problems, and finding the consumption path.

In the future, we will discuss the calculus of variations for the high-order and multivariable problem with $\nabla$-derivatives of exponential function, and their applications in scientific fields.

Acknowledgment. This work was supported by National Natural Science Foundation of China (Grant No.11501274), the Fundamental Research Funds for the Central Universities (Grant No. 2412017FZ004). 


\section{References}

[1] R. Agarwal, M. Bohner, D. O'Regan and A. Peterson, Dynamic equations on time scales: a survey, J. Comput. Appl. Math. 141 (1), 1-26, 2002.

[2] C.D. Ahlbrandt, M. Bohner and J. Ridenhour, Hamiltonian systems on time scales, J. Math. Anal. Appl. 250 (2), 561-578, 2000.

[3] R. Almeida and D.F. Torres, Isoperimetric problems on time scales with nabla derivatives, J. Vib. Control, 15 (6), 951-958, 2009.

[4] F.M. Atici, D.C. Biles and A. Lebedinsky, An application of time scales to economics, Math. Comput. Model. 43 (7-8), 718-726, 2006.

[5] Z. Bartosiewicz and E. Pawluszewicz, Realizations of nonlinear control systems on time scales, IEEE Trans. Autom. Control, 53 (2), 571-575, 2008.

[6] Z. Bartosiewicz and E. Pawluszewicz, Dynamic feedback equivalence of time-variant control systems on homogeneous time scales, Int. J. Math. Stat. 5 (A09), 11-20, 2009.

[7] Z. Bartosiewicz, N. Martins and D.F. Torres, The second euler-lagrange equation of variational calculus on time scales, Eur. J. Control, 17 (1), 9-18, 2011.

[8] Z. Bartosiewicz, E. Piotrowska and M. Wyrwas, Stability, stabilization and observers of linear control systems on time scales, Proc. IEEE Conf. on Decision and Control, New Orleans, LA, USA, 2803-2808, 2007.

[9] Z. Bartosiewicz, U. Kotta, E. Pawluszewicz and M. Wyrwas, Algebraic formalism of differ-ential one-forms for nonlinear control systems on time scales, Proc. Est. Acad. Sci. 56 (3), 264-282, 2007.

[10] M. Bohner, Calculus of variations on time scales, Dynam. Syst. Appl. 13 (3-4), 339349, 2004.

[11] M. Bohner and A. Peterson, Dynamic equations on time scales: An introduction with applications, Birkhauser Boston, Boston, MA, 2001

[12] J.J. DaCunha, Stability for time varying linear dynamic systems on time scales, J. Comput. Appl. Math. 176 (2), 381-410, 2005.

[13] R. David, Advanced macroeconomics, McGraw-Hill, Irwin, 2011.

[14] R.A. Ferreira and D.F. Torres, Remarks on the calculus of variations on time scales, Int. J. Ecol. Econ. Stat. 9 (F07), 65-73, 2007.

[15] R.A. Ferreira and D.F. Torres, Higher-order calculus of variations on time scales, in: Mathematical Control Theory and Finance, Springer, Berlin, 136 (1), 149-159, 2008.

[16] M. Guzowska, A.B. Malinowska and M.R.S. Ammi, Calculus of variations on time scales: applications to economic models, Adv. Differ. Equ. 2015 (1), 203, 2015.

[17] R. Hilscher and V. Zeidan, Calculus of variations on time scales: weak local piecewise crd1 solutions with variable endpoints, J. Math. Anal. Appl. 289 (1), 143-166, 2004.

[18] R. Hilscher and V. Zeidan, Weak maximum principle and accessory problem for control problems on time scales, Nonlinear Anal. TMA, 70 (9), 3209-3226, 2009.

[19] A.B. Malinowska and D.F. Torres, Necessary and sufficient conditions for local pareto optimality on time scales, J. Math. Sci. (NY), 161 (6), 803-810, 2009.

[20] A.B. Malinowska and D.F. Torres, Strong minimizers of the calculus of variations on time scales and the weierstrass condition, P. Est. Acad. Sci. 58 (4), 205-212, 2009.

[21] N. Martins and D.F. Torres, Calculus of variations on time scales with nabla derivatives, Nonlinear Anal. TMA, 71 (12), 763-773, 2009.

[22] D. Mozyrska and Z. Bartosiewicz, Observability of a class of linear dynamic infinite systems on time scales, Proc. Est. Acad. Sci. 56 (4), 347-358, 2007.

[23] J. Seiffertt, S. Sanyal and D.C. Wunsch, Hamilton-Jacobi-Bellman equations and approximate dynamic programming on time scales, IEEE Trans. Syst. Man Cybern. Part B: Cybern. 38 (4), 918-923, 2008.

[24] Z. Zhan, W. Wei and H. Xu, Hamilton-Jacobi-Bellman equations on time scales, Math. Comput. Model. 49 (9-10), 2019-2028, 2009. 